\title{
Axenfeld-Rieger syndrome resulting from mutation of the FKHL7 gene on chromosome 6p25
}

\author{
Farideh Mirzayans ${ }^{1}$, Douglas B Gould ${ }^{1}$, E Héon², Gail D Billingsley², Jason C Cheung ${ }^{2}$, \\ Alan J Mears ${ }^{1}$ and Michael A Walter ${ }^{1}$
}

\author{
${ }^{1}$ Departments of Ophthalmology and Medical Genetics, University of Alberta, Edmonton, AB; ${ }^{2}$ Eye Research Institute \\ of Canada, University of Toronto, Toronto, ON, Canada
}

\begin{abstract}
Mutations in the forkhead-like 7 (FKHL7) gene have been recently shown to cause juvenile glaucoma and anterior segment anomalies. We report on a three-generation family with Axenfeld-Rieger syndrome (ARS), harboring an alteration in the FKHL7 gene. Genetic linkage analyses excluded the ARS phenotype from chromosomes 4q25 and 13q14, the locations of the PITX2 and RIEG2 loci, respectively. Evidence of linkage was observed with markers at 6p25, near the FKHL7 gene. Direct sequencing of FKHL7 detected a C67T mutation that segregated with the ARS phenotype in this family, but was not detected in over 80 control chromosomes. This mutation is predicted to cause a nonsense mutation of the FKHL7 protein (GIn23Stop) upstream of the forkhead DNA-binding domain, and thus to generate a truncated FKHL7 protein product. This discovery broadly implicates FKHL7 in ocular, craniofacial, dental, and umbilical development. European Journal of Human Genetics (2000) 8, 71-74.
\end{abstract}

Keywords: Axenfeld-Rieger syndrome; forkhead-like 7; FKHL7; PITX2; Axenfeld-Rieger anomaly; chromosome6p25; mutation

\section{Introduction}

Axenfeld-Rieger syndrome (ARS; OMIM No 180500) is an autosomal dominantly inherited developmental disorder in which patients present with ocular and non-ocular clinical findings. ${ }^{1}$ Ocular characteristics include hypoplasia of the anterior iris stroma, microcornea, anterior chamber synechiae, corneal opacity, and juvenile onset glaucoma. Nonocular features typically include maxillary hypoplasia, hypodontia, and failure of involution of the periumbilical skin. Patients with the autosomal dominantly-inherited AxenfeldRieger anomaly (ARA) present with the characteristic ocular features alone.

Various chromosomal aberrations have been found in association with ARS, including the involvement of chromosomes 4, 6, 9, 13, 18, and 21 (reviewed by Nielsen and colleagues $^{2}$ ). Two genes underlying the ARS phenotype have been local ized to chromosomal regions, the first to $4 q 25^{3}$ and

Correspondence: Michael A Walter, Ocular Genetics Laboratory, 832 Medical Sciences Building, University of Alberta, Edmonton, Alberta, Canada. Tel: +1403492 3028; Fax: +1403492 6934; E-mail: mwalter@ualberta.ca

Current address: WK Kellogg Eye Center, Ann Arbor, MI, USA

Received 15 January 1999; revised 1 April 1999; accepted 12 April 1999 the second to $13 q 14,{ }^{4}$ indicating that ARS is genetically heterogeneous.

Mutations of the PITX 2 gene have been shown to underlie the ARS phenotype in ARS families in which the disease phenotype is linked to $4 \mathrm{q} 25 .^{5}$ PITX2 is a developmental control gene of the bicoid homeobox gene family. Interestingly, the phenotype in two families with iridogoniodysgenesis syndrome (IRID2; OMIM No 137600), an autosomal dominant condition in which patients present with iris stromal hypoplasia, goniodysgenesis, abnormal angle vascularity, and ARS-like non-ocular findings, have been shown to result from different missense mutations of the PITX2 gene. ${ }^{6,7}$ To date, $10-30 \%$ of ARS patients have been found to have mutations of the PITX 2 gene located at chromosome $4 q 25^{5}$ (al so Kulak and Walter, un published data, 1999).

Genetic linkage analysis localized a gene underlying ARA to chromosome $6 \mathrm{p} 25 .^{8}$ Independent results from our laboratory $^{9}$ and that of Sheffield, ${ }^{10}$ demonstrated that ARA mapping to $6 \mathrm{p} 25$ results from mutations of the forkheadlike7 (FKHL7) gene. FKHL7 is a member of the forkhead transcription factor gene family shown to be involved in embryonic development, tissue-specific gene expression, and oncogenesis. ${ }^{11-15}$ Homozygous null mutations of $M f 1$, the 
a

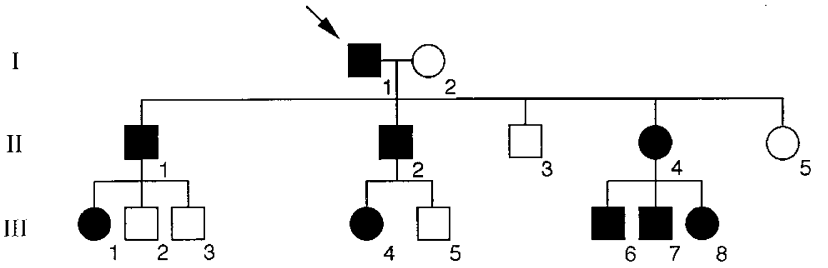

b

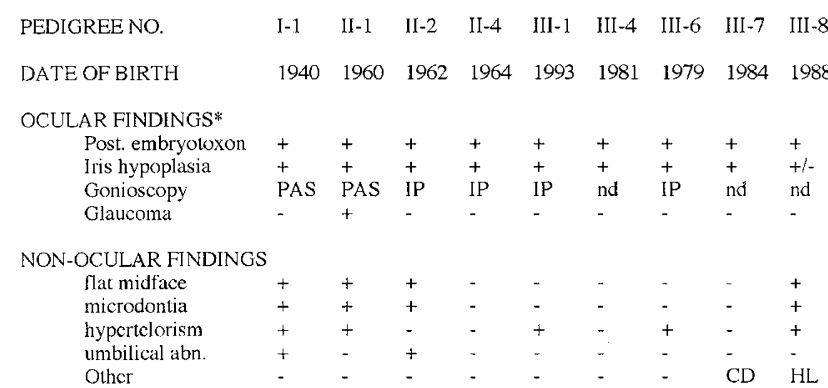

Figure 1 Three-generation family with nine members affected with Axenfeld-Rieger syndrome (ARS). a Pedigree of the family with ARS. Affected individuals are indicated by filled symbols, unaffected by unfilled symbols. The proband, affected individual $\mathrm{I}-1$, is indicated by an arrow. b Clinical findings in *family members affected with ARS; post; posterior; PAS: peripheral anterior synchiae; IP: iris processes; nd: not done; abn: abnormalities including failure of involution; CD: cardiac defect; HL: hearing loss.

murine homologue of FKHL7, have been shown to be responsible for the congenital hydrocephalus (ch) phenotype in mice. ${ }^{16}$ These results suggest that FKHL 7 has a key role in the development of the mammalian eye and brain.

We report here on a three-generation family with ARS, harboring a novel nonsense mutation in the FKHL 7 coding sequence, upstream of the FKHL 7 DNA-binding domain. This discovery not only indicates that mutations of FKHL7 can result in both ARA and ARS, but also broadly implicates FKHL7 in ocular, craniofacial, dental, and umbilical development.

\section{Materials and methods \\ Subjects}

The family with ARS studied in this report had nine affected individuals in three generations (Figure 1). Affected individuals presented with a variable degree of iris hypoplasia, displaced pupils (corectopia), and a prominent, anteriorlydisplaced Schwalbe's line (posterior embryotoxon) to which peripheral iris strands were attached bridging the iridocorneal angle (Figure2). Glaucoma was observed in individual II-1. A summary of the clinical findings in all affected

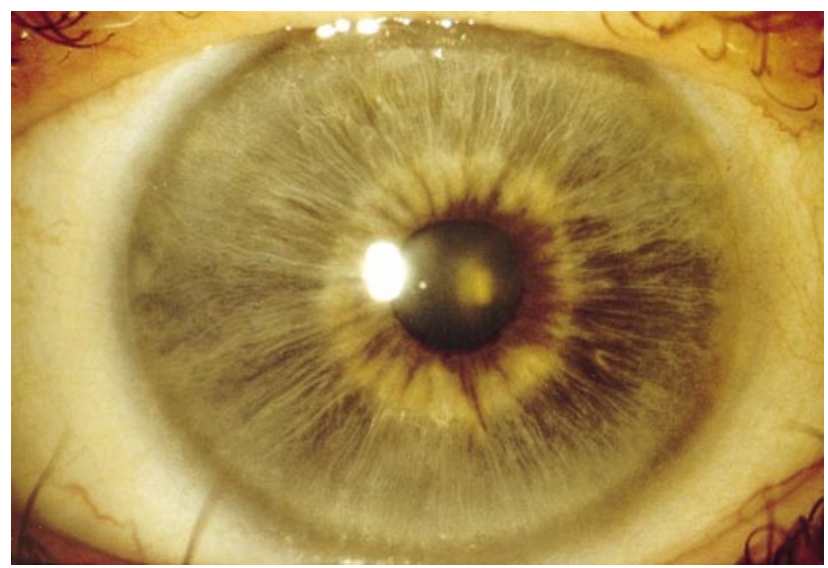

Figure 2 Photograph of the left eye of the proband (I-1) affected with ARS, displaying diffuse iris hypoplasia and posterior embryotoxon.

individuals is presented in Figure 1b. The study and collection of blood samples from all individuals included in this report were approved by the Toronto Hospital Committee for Research on Human Subjects.

\section{Genotypic and molecular studies}

Blood samples were collected in EDTA tubes and DNA prepared from isolated white blood cells as described. ${ }^{17}$ Oligonucleotide primers for microsatellite markers were obtained from Research Genetics (Huntsville, AL, USA) and ACGT (Toronto, ON, Canada). PCR primers and conditions for the amplification of the FKHL7 gene were as described. ${ }^{9}$ PCR amplification for linkage and SSCP analyses were performed as described. ${ }^{18}$ PCR products for linkage analysis were separated upon $6 \%$ denaturing polyacrylamide gels. SSCP analysis was conducted on $6 \%$ acrylamide non-denaturing gels containing $7 \%$ glycerol, with electrophoresis at $60 \mathrm{~W}$ at $4^{\circ} \mathrm{C}$. After electrophoresis, gels were dried and used for autoradiography. DNA sequencing was done manually using a ${ }^{33}$ P-radiolabelled terminator thermosequenase cycle sequencing kit from Amersham-Pharmacia (Baie d'Urfé, PQ, Canada). All PCR products were purified using Qiagen columns (Mississanga, ON, Canada), prior to sequencing.

\section{Linkage analysis}

Linkage analysis was conducted with a DOS-compatible Power Macintosh 6100/66 computer. Lod score values were calculated using the M LINK option of the LINKAGE program. Analysis was conducted under the assumption that ARA is a fully penetrant autosomal dominant disease with a mutation rate of $10^{-6}$, and a gene frequency in the population of 0.0001 . The allele frequencies were assumed to be equal for the markers used in this study. 


\section{Results}

\section{Linkage studies}

Linkage of the ARS phenotype in this family to the PITX 2 locus at $4 q 25$ was excluded by examination of three polymorphic markers in the 4q25 region, D4S3240, D4S2623, and D4S406 (data not shown). Examination of six polymorphic markers (D13S894, D13S1288, D13S1253, D13S1248, D13S263, and D13S325) spanning the RIEG2 critical region at $13 q 14^{4}$ was consistent with exclusion of the RIEG2 gene (data not shown). In contrast, complete linkage of ARS in this family to polymorphic loci flanking the FKHL7 gene at chromosome $6 \mathrm{p} 25$ was found. A maximum lod score $\left(Z_{\max }\right)$ of 2.71 at a recombination fraction $(\theta)$ of 0.00 was obtained with marker D6S344 located at the distal short arm of chromosome 6 (6p25). D6S344 is located approximately $10 \mathrm{~kb}$ from the FKHL 7 gene (Gould and Walter, unpublished data). Positive lod scores of 2.41 at $\theta$ of 0.00 were also found with D6S1600 and D6S967 also located at 6p25. Whilst the small size of the ARS family together with only partially informative markers precluded the $Z_{\max }$ lod scores from reaching the critical value of 3.00 required to indicate significant linkage, this suggestion of possible linkage to polymorphic markers flanking the FKHL 7 gene was sufficient to initiate screening of the FKHL 7 gene for an ARS-associated mutation.

\section{Mutation of the FKHL7 gene associated with ARS}

FKHL7 primer sets were used to PCR amplify the single exon FKHL7 gene in affected individual (I-1) to identify the mutation of the FKHL7 gene. Direct DNA sequencing of the FKHL7 gene in DNA from I-1 revealed a C67T alteration within one allele of FKHL7 (Figure 3). SSCP analysis indicated that this nucleotide alteration segregated with the disease phenotype in this family, but was not found in over 80 normal chromosomes (data not shown). This mutation is predicted to result in a GIn23Stop nonsense mutation prior to the FKLH7 forkhead domain.

\section{Discussion}

To date, over 80 forkhead family genes have been cloned and characterized. There is increasing evidence that members of the forkhead/winged helix gene family play key roles in embryonic development, cell fate determination, tumorigenesis, and tissue-specific gene expression in both vertebrates. ${ }^{15}$ Recently, mutations of the thyroid transcription factor 2/forkhead-like15 (FKHL15) and forkhead-like7 (FKHL7) genes have been found to cause diseases - thyroid agenesis, cleft palate, and choanal atresia, ${ }^{19}$ and AxenfeldRieger anomaly, ${ }^{9,10}$ respectively.

We studied a family with nine members affected with ARS, exhibiting varying degrees of iris hypoplasia, posterior embryotoxon, and iris strands, in addition to non-ocular findings including umbilical anomalies, flat midfaces, and small teeth. Linkage analyses excluded the region that

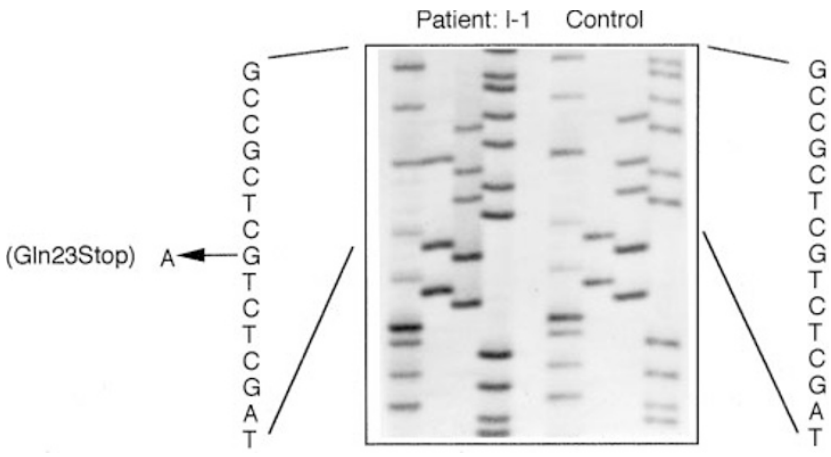

Figure 3 DNA sequence analysis indicating an ARS-associated mutation in the FKHL7 gene. Autoradiographs showing sequence analysis of the mutation identified in the ARS family. PCR products were amplified using primer set $C^{9}$ from DNA samples of individual I-1 and by a non-affected control individual, and were directly sequenced. Normal control sequence is shown on the right; sequence from affected individual I-1 is shown on the left. The DNA sequence from the reverse primer is shown in each case, with the lanes representing bases GATC from left to right. Position of the mutation is shown to the left. The predicted effect of the FKHL7 mutation is indicated to the far left. Pedigree number is as in Figure1.

contains the PITX2 gene at $4 q 25$ and the RIEG2 minimal critical interval at 13q14 from underlying ARS in this family. Linkage analysis, however, indicated possible linkage of ARS in this family to markersflanking the $6 \mathrm{p} 25$ region containing the FKHL 7 gene. Direct DNA sequencing of the FKHL7 gene in DNA from this family revealed a C67T alteration that segregated with the disease phenotype. This mutation, predicted to result in a GIn23Stop nonsense mutation upstream of the FKHL7 forkhead domain (Figure 3), was not found in over 80 normal chromosomes.

This report is the first description of a FKHL7 mutation associated with ARS. This work supports the increasing evidence that FKHL7 has a significant role in the development of the anterior segment of the eye and brain. Mice with homozygous null alleles of Mf1, the murine homologue of FKHL7, die at birth with hydrocephalus, heart defects, eye anomalies, and multiple skeletal defects. ${ }^{16}$ Mutations have been identified in the FKHL7 coding region in several small families affected with ARA. ${ }^{9,10}$ All of the mutations reported to date in ARA patients either have been upstream of the FKHL7 forkhead DNA-binding domain and are predicted to result in premature protein truncation prior to the forkhead domain, or have resulted in substitutions of conserved amino acids within the forkhead DNA-binding domain. The FKHL7 mutation described here (GIn23Stop), and the Mf1 mutation underlying the ch phenotype in the mouse (GIn123Stop), are the only nonsense mutations described to date in the FKHL7/ Mf1 gene. Both the ARS and ch phenotypes involve nonocular tissues, unlike ARA. It is possible that the FKHL7 mutations associated with the clinically less severe ARA 
phenotype could result from retention of residual FKHL7 activity in the cases of FKHL7 missense mutations, or from low level reinitiation of translation of the FKHL 7 mRNA from downstream inframe ATG codons in the two cases of FKHL 7 frameshift mutations. Interestingly, a $4.0 \mathrm{~kb}$ alternative transcript, smaller than the $4.5 \mathrm{~kb}$ FKHL 7 transcript observed in all other tissues examined, has been observed in fetal kidney, indicating that alternative FKHL7 transcripts can also be produced in some tissues. ${ }^{9,10,12}$ It remains to be determined if such alternative FKHL7 transcripts have a role in the development of the non-ocular dental, maxillary, and umbilical systems affected in patients with ARS. The nonsense mutation of FKHL7 found in the family reported here indicates that haploinsufficiency of FKHL7 could underlie the more clinically severe phenotype present in ARS patients.

To date, only $10-30 \%$ of ARS patients have been found to have mutations of the PITX2 gene located at chromosome $4 \mathrm{q} 25^{5}$ (also Kulak and Walter, unpublished data). Our finding of an ARS-associated FKHL7 mutation raises the possibility that a significant proportion of ARS patients could have FKHL7 mutations. In addition, the finding of ARSassociated FKHL7 mutations expands the role of FKHL7 in mammalian embryogenesis, implicating FKHL7 in ocular, craniofacial, dental, and umbilical differentiation, as well as in developmental glaucoma.

\section{Acknowledgements}

We would like to express our gratitude to the ARS family for their co-operation and their enthusiasm for this project. We thank the members of the Departments of M edical Genetics and Ophthalmology, University of Alberta, for critical comments on this research and manuscript. This research was funded by grants from the Alberta Heritage Fund for Medical Research (AHFMR grant EG9400216 to MAW), the Medical Research Council of Canada (MRC Grant MT12916 to MAW), the Weston Foundation (to EH), and from Physicians and Surgeons Incorporated (grant PSI 97-02 to EH). DBG is the recipient of a MRC doctoral studentship. MAW is a MRC and AHFMR scholar. AJM was funded by an AHFMR postdoctoral fellowship.

\section{References}

1 Alward LM, Murray JC: Axenfeld-Rieger Syndrome. In: Wiggs J (ed). Molecular Genetics of Ocular Disease. Wiley-Liss: New York, NY, 1995, 31-50.

2 Nielsen F, Tranebjaerg L: A case of partial monosomy $21 q 22.2$ associated with Rieger's syndrome. J Med Genet 1984; 21: 218-221.
3 Murray JC, Bennett SR, Kwitek AE et al: Linkage of Rieger syndrome to the region of the epidermal growth factor gene on chromosome4. Nat Genet 1992; 2: 46-49.

4 PhillipsJC, Del Bono EA, Haines JL et al: A second locus for Rieger Syndrome maps to chromosome13q14. Am J Hum Genet 1996; 59: 613-619.

5 Semina EV, Reiter R, Leysens NJ et al: Cloning and characterization of a novel bicoid-related homeobox transcription factor gene, RIEG, involved in Rieger syndrome. Nat Genet 1996; 14: 392-399.

6 Alward WL, Semina EV, Kalenak W et al: Autosomal dominant iris hypoplasia is caused by a mutation in the Rieger Syndrome (RIEG/ PITX2) gene. Am J Ophthalmol 1998; 125: 98-100.

7 Kulak SC, Kozlowski K, Semina EV, Pearce WG, Walter MA: Mutation in the RIEGl gene in patients with iridogoniodysgenesis syndrome. Hum Mol Genet 1998; 7: 1113-1117.

8 Gould DB, Mears AJ, Pearce WG, Walter MA: Autosomal dominant Axenfeld-Rieger anomaly maps to $6 \mathrm{p} 25$. Am J Hum Genet 1997; 61: 765-768.

9 Mears AJ, Jordan T, Mirzayans F et al: Mutations of the forkhead/ winged helix gene, FKHL7, in patients with Axenfeld-Rieger Anomaly. Am J Hum Genet 1998; 63: 1316-1328.

10 Nishimura DY, Swiderski RE, Alward LM et al: The forkhead transcription factor gene FKHL7 is responsible for glaucoma phenotypes which map to 6p25. Nat Genet 1998; 19: 140-147.

11 Shapiro DN, Sublett JE, Li BT, Downing JR, Naeve CW: Fusion of pax3 to a member of the forkhead family of transcription factors in human alveolar rhabdomyosarcoma. Cancer Res 1993; 53: 5108-5112.

12 Pierrou S, Hellqvist M, Samuelsson L, Enerback S, Carlsson P: Cloning and characterization of seven human forkhead proteins: binding site specificity and DNA bending. EMBO J 1994; 13: 5002-5012.

13 Sasaki H, Hogan B: Hnf-3-beta as a regulator of floor plate development. Cell 1994; 76: 103-115.

14 Larsson C, Hellqvist M, Pierrou S, White I, Enerbäck S, Carlsson P: Chromosomal localization of six human forkhead genes, freac-I (FKHL5), -3 (FKHL7), -4 (FKHL8), -5 (FKHL9), -6 (FKHL10), and -8 (FKHL12). Genomics 1995; 30: 464-469.

15 Kaufmann E, Knochel W: Five years on the wings of fork head. Mech Devel 1996; 57: 3-20.

16 Kume T, Deng K-Y, Winfrey V, Gould DB, Walter MA, Hogan BLM : The forkhead/winged helix gene Mf1 Is disrupted in the pleiotropic mouse mutation congenital hydrocephalus. Cell 1998; 93: 985-996.

17 Miller SA, Dykes DD, Polesky HF: A simple salting out procedure for extracting DNA from human nucleated cells. Nucl Acid Res 1988; 16: 1215 .

18 Mirzayans F, Pearce WG, MacDonald IM, Walter MA: Mutation of the PAX 6 gene in patients with autosomal dominant keratitis. Am J Hum Genet 1995; 57: 539-548.

19 Clifton-Bligh RJ, Wentworth JM, Heinz P et al: Mutation of the gene encoding human TTF-2 associated with thyroid agenesis, cleft palate and choanal atresia. Nat Genet 1998; 19: 399-401. 